\title{
Simulating and quantifying legacy topographic data uncertainty: an initial step to advancing topographic change analyses
}

\author{
Thad Wasklewicz ${ }^{1 *}$, Zhen Zhu² and Paul Gares ${ }^{1}$
}

\begin{abstract}
Rapid technological advances, sustained funding, and a greater recognition of the value of topographic data have helped develop an increasing archive of topographic data sources. Advances in basic and applied research related to Earth surface changes require researchers to integrate recent high-resolution topography (HRT) data with the legacy datasets. Several technical challenges and data uncertainty issues persist to date when integrating legacy datasets with more recent HRT data. The disparate data sources required to extend the topographic record back in time are often stored in formats that are not readily compatible with more recent HRT data. Legacy data may also contain unknown error or unreported error that make accounting for data uncertainty difficult. There are also cases of known deficiencies in legacy datasets, which can significantly bias results. Finally, scientists are faced with the daunting challenge of definitively deriving the extent to which a landform or landscape has or will continue to change in response natural and/or anthropogenic processes. Here, we examine the question: how do we evaluate and portray data uncertainty from the varied topographic legacy sources and combine this uncertainty with current spatial data collection techniques to detect meaningful topographic changes? We view topographic uncertainty as a stochastic process that takes into consideration spatial and temporal variations from a numerical simulation and physical modeling experiment. The numerical simulation incorporates numerous topographic data sources typically found across a range of legacy data to present high-resolution data, while the physical model focuses on more recent HRT data acquisition techniques. Elevation uncertainties observed from anchor points in the digital terrain models are modeled using "states" in a stochastic estimator. Stochastic estimators trace the temporal evolution of the uncertainties and are natively capable of incorporating sensor measurements observed at various times in history. The geometric relationship between the anchor point and the sensor measurement can be approximated via spatial correlation even when a sensor does not directly observe an anchor point. Findings from a numerical simulation indicate the estimated error coincides with the actual error using certain sensors (Kinematic GNSS, ALS, TLS, and SfM-MVS). Data from 2D imagery and static GNSS did not perform as well at the time the sensor is integrated into estimator largely as a result of the low density of data added from these sources. The estimator provides a history of DEM estimation as well as the uncertainties and cross correlations observed on anchor points. Our work provides preliminary evidence that our approach is valid for integrating legacy data with HRT and warrants further exploration and field validation.
\end{abstract}

Keywords: Stochastic models, Digital terrain models, Legacy data, Geomorphic change detection, High-resolution topography

\footnotetext{
* Correspondence: wasklewiczt@ecu.edu

'Department of Geography, Planning, and Environment, East Carolina

University, Brewster Building A227, Greenville, NC 27858, USA

Full list of author information is available at the end of the article
} 


\section{Introduction}

Topographic data sources are an essential source of information used to not only address scientific questions related to landscape evolution, but also to inform policy makers and land managers of recent environmental changes. Earth scientists have also begun to carve out a role in the global climate agenda (Lane 2013) and topographic data will play a significant role in these types of analyses. Recent examples of this research avenue place greater emphasis on understanding how humans modify the physical environment (Tarolli and Sofia 2016) as well as examining the likelihood of extreme weather leading to magnitude and frequency changes in geomorphic processes and surficial features (Spencer et al. 2017). These two recent research trends also highlight a need to be able to examine recent topographic changes in the context of longer-term rates of change. This task will require greater reliance on integrating legacy and more recent high-resolution topography (HRT) data sources to aid in our understanding of environmental change (Glennie et al. 2014; Pelletier et al., 2015). However, the integration of disparate topographic data sources introduces numerous opportunities to increase uncertainty in measurements through space and time. Herein, we provide a means to assay and portray data uncertainty when fusing legacy and recent HRT data to assess change in landforms. Statistic and stochastic models are employed that are not only consistent with topographic data structures simulated in this research, but also can track the correlation of errors over time and across different data sources to establish reliable detection levels. Our findings represent an initial step to providing researchers with the ability to detect topographic changes with a degree of certainty.

Topographic legacy datasets come from a variety of field and remotely sensed sampling techniques and instrumentation (Wasklewicz et al. 2013). Data quality, resolution, and temporal availability from these disparate sources have varied significantly over their historical development. Current research challenges in earth science rely on a deeper understanding of how to integrate these varied datasets and their associated data uncertainty. Advancement of scientific research on landscape evolution and environmental change detection rely on definitively measuring how the changing earth surface. More exacting measures would aid in items such as the development of more precise early warning systems where landscape dynamics pose hazards and risks to society, and could lead to potential innovations in the design of infrastructure that is more resilient to dynamic surficial processes.

Research focused on topographic changes has relied heavily on pre-event topographic data sets (e.g., Wasklewicz and Hattanji 2009; Wheaton et al. 2010).
Some studies use the same instrumentation and apply a consistent methodology throughout the observation period (e.g., Wester et al. 2014; Staley et al. 2014; Wasklewicz and Scheinert 2015). The integration of topographic data from a single source with the aid of a repeatable surveying campaign over time has presented an opportunity to reduce the systematic errors while accounting for other positional errors and surface representation uncertainties. However, when repeatable surveying campaigns are not followed, researchers found data can possess erroneous calibration and improper error modeling (Oskin et al. 2012; Glennie et al. 2014). These inherent issues are expressed as substantial systematic errors, which lead to improper measurements when compared with the post-event data. Glennie et al. (2014) warn systematic errors must be minimized or removed prior to differencing the pre- and post-event airborne laser scanning (ALS) data sets. Schaffrath et al. (2015) identified comparable issues with both vertical and horizontal measures from pre- and post-flood ALS data being inadequately defined from the use of different geoid models and poor co-registration of flight lines, respectively.

The complexity of the data uncertainty increases with the incorporation of legacy data. A single instrument is never used in these cases. Instead, researchers attempt to fuse topographic data from multiple instruments as technological advances in data collection have occurred over time (Crowell et al. 1991; Carley et al. 2012; Schaffrath et al. 2015). A variety of topographic data sources that include contour maps, cross-sections or topographic profiles, raster, triangulated irregular networks (TINs), and point clouds can be integrated as legacy data into these types of analyses. Each different data source introduces varying: data quality, spatial resolution, and temporal consistency. These items increase the spatially variable uncertainty of the measurements taken during analyses of these disparate data sets, which must be accounted for in the presentation of the findings.

The recent incorporation of SfM-MVS (structure from Motion-Multi-View Stereo) techniques has the benefit of permitting researchers to extend back further the legacy record by using archived aerial photographs to reconstruct a point cloud and digital elevation model (DEM) (Gomez et al. 2015; Micheletti et al. 2015), but also adding further complications to examining spatially variable uncertainty. While all signs from these studies indicate a strong potential to use these data sources to measure topographic changes from legacy data, several issues must be addressed to accurately use this approach. Some of the common photogrammetric issues included inconsistent image quality, varied scales, objects in motion, clouds, and other superfluous information in the photos (Gomez et al. 2015; Micheletti et al. 2015). Initial results 
indicated aerial imagery at a scale of 1:20,000 may only be able to detect changes in the 1 to $1.5 \mathrm{~m}$ range with quality ground control points (Micheletti et al. 2015), but in some instances this value can be at a coarserscale (Fonstad et al. 2013; Gomez et al. 2015). Another major concern with applying this technique is overestimation of the topography within areas of vegetation and locations where there is disparate topographic relief (Gomez et al. 2015; Micheletti et al. 2015). However, some these obstacles can be overcome with the appropriate establishment of ground control points and consistent field validation of the results where possible and others will require development of uncertainty measures capable of uncovering errors in complex topography.

Robust estimates of spatially variable uncertainty have received more recent attention, but remain in their infancy (Schaffrath et al. 2015). As highlighted in Carley et al. (2012), researchers have generally used three different approaches when examining uncertainty in topographic legacy data: (1) a uniform error threshold (Brasington et al. 2003; Lane et al. 2003; Milan et al., 2007), which have been noted to bias data in various topographic sequences; (2) spatial variance thresholds used to produce a minimum level of detection raster file (Heritage et al. 2009; Milan et al. 2011), which work well in settings with high-density point clouds and mid- to fine-scale roughness features; and (3) error-source threshold methods developed from fuzzy inference systems (Wheaton et al. 2010, Schaffrath et al. 2015), which has been proven to assess the spatial variability of uncertainty often inherent in the digital elevation models (DEM[s]) used in topographic changes. Carley et al. (2012) employed a hybrid method of the spatial variance approach (\#2) to consider the addition of legacy topographic map data to their analyses. They applied a survey and interpolation error (SIE) equation (Heritage et al. 2009) to produce SIE point grids for the DEMs used in the DEM-of-difference and combined these to produce a critical error threshold (Brasington et al. 2000, 2003; Lane et al. 2003) for each cell and a level-of-detection surface was generated from this style of analysis.

The issue of incorporating additional measurements with existing models or maps has also been encountered in the robotics and computer vision communities. For example, a robotic mapping problem can be solved by using discretized map representation. A common approach of mapping a $2 \mathrm{D}$ or $3 \mathrm{D}$ space is to abstract the whole space into a list of objects with corresponding properties, such as location and uncertainties (Thrun et al. 2005). For example, a 2D space can be generalized into an "occupancy grid" (Elfe 1989). The occupancy grid represents a dense 3D map with a finite array of points. Any additional measurements or data sources over the same space can then relate to one or several grid points. A similar concept of sparse discretized mapping has also been applied into our approach to model topographic features. A 3D surface in space can be approximated using a finite set of known points, on which any given point can be predicted via a wide variety of interpolation techniques. Some of these techniques are commonly used in geosciences, including Kriging models (Krige 1951) and other types of regression methods (Williams 1998; Dumitru et al. 2013).

There is also a realization that it is equally important, if not more so, to accurately represent the uncertainty function across the whole space. Spatial uncertainty modeling is a key element in the regression-based prediction processes. Moreover, the spatial uncertainty functions may change over time due to the underlying geomorphic processes, and this can be evaluated with a stochastic estimator, such as a Kalman filter (Kalman 1960). For example, Mardia et al. (1998) and Cortes (2009) suggested the combination of spatial and temporal modeling by using Kriging and Kalman filters.

Here, our focus is on the spatio-temporal uncertainty model itself, instead of any specific interpolation method. Our approach produces an efficient, accurate, and robust uncertainty model that opens the door to the integration of legacy data and new sensors, and provides more definitive measures of landform and landscape evolution from a variety of sources. A major benefit and advance of our adopted approach is that an optimum interpolation method can be applied to this model, which would estimate or predict the elevation and the associated uncertainty for any location in the entire region, at any given time in history or even in the future.

\section{Methods/Experimental}

\section{Spatio-temporal uncertainty model}

Here, we consider topographic uncertainty as a stochastic process that takes into consideration spatial and temporal variations. The spatial uncertainty can be modeled as a Gaussian process, which tends to vary across the region of interest, and yet often has local correlations. A higher level of spatial correlation may be expected from smooth surfaces, whereas sudden elevation changes, such as a steep cliff, will result in lower correlation. A covariance function can describe this type of uncertainty model and is required by most interpolation or prediction techniques. Although it could be challenging to obtain the complete covariance function, especially if such function is also changing over time, following Williams (1998), we estimate a covariance matrix over a finite set of hypothetical anchor points. The covariance matrix is updated over time, based on input from a variety of sensors, such as laser scanners and global navigation satellite system (GNSS) survey. Although the filter does 
benefit from direct observations whenever available, sensors are not expected to make direct observation of the anchor points. For example, a terrestrial laser scanner (TLS) provides a high-resolution scan of the region, but the point cloud is not guaranteed to overlap the hypothetical anchor points. Static GNSS surveys in historical data, on the other hand, may only be available on a few points in a region, which are even less likely to overlap with the anchor points. The lack of coincident data with the anchor does not preclude the use of this method, rather this method does not require any direct sensor observations in our experimental design or in any future field applications of this methodology.

Anchor point distributions are instead dependent upon the complexity of the topographical features, which is independent of any specific sensor measurements. However, sensor observation made at any given location within the region can still be used to update the uncertainty model of the neighboring anchor point(s). To achieve that, we further assume that more points will be used to model an area with lower spatial correlation, such that the elevation at a given location can be interpolated or extrapolated with neighboring anchors with sufficient accuracy. Thus, a surficial feature with topographic complexity is covered with densely populated anchor points, whereas less topographically complex areas are covered with a sparse scatter of anchor points.

The uncertainty model and associated anchor points not only account for the spatial uncertainty, but also consider the function of time. A stochastic estimator traces the evolution of such randomness, in which the anchor points form a space of "states". Thus, the elevation and uncertainty of any given point at any time can be approximated with a combination of these states. In a software simulation, we created a landform of 150 by $150 \mathrm{~m}$, and simulated the elevation change over the course of 30 years. Both the landform and the elevation change have non-linear surfaces (Fig. 1). To demonstrate the concept of using anchor points, we first formulated a 5 by 5 array, which has sufficient density for a discretized representation of the simulated landform. However, anchor points do not necessarily form any regular shape in general.

Data from a TLS, an ALS, a 2D aerial camera, a stereo vision system, structure from motion SfM-MVS from a limited number of historic aerial photographs taken from a nadir-looking camera, static and Kinematic GNSS surveys are considered in our simulations (Table 1). These sensors differ greatly in accuracy and resolution. We assume all the sensor errors can be projected onto the vertical direction, and thus only the vertical errors are of concern in developing an objective comparison among the various sensors.

Gaussian error models are used for GNSS positioning in this simulation. Naturally, the static surveys are more accurate. However, the Kinematic GNSS survey will gather more data points (Table 1).

The uncertainty in laser ranging measurements can be modeled with two main components, angular and radial. Although the complete ranging error model may be sophisticated (Thrun et al. 2005), it is often sufficient to assume that in practice, both error sources follow a normal distribution. The underlying mechanisms are independent from each other (Glennie 2007). Figure 2 illustrates a typical application of laser scanner, which applies to both TLS and ALS. The laser scanner is elevated from the ground by $h$, and it scans a location on the ground at a line of sight distance $r$. Errors in the horizontal (azimuth) and vertical (elevation) angles result from laser beam width and the precision in orientation measurements and follow a 2D normal distribution (red ellipse in Fig. 2). Since the focus of this work is not on the high-fidelity simulation of ALS data, the comprehensive error model (Glennie 2007) is abstracted and represented with two components: a radial error $\sigma_{n}$ and a vertical angular error $\sigma_{\theta}$. A target observed at distance $r$ has the total uncertainty of $\sigma_{v}$. Compared to an ALS, a TLS is closer to the ground, produces a denser point
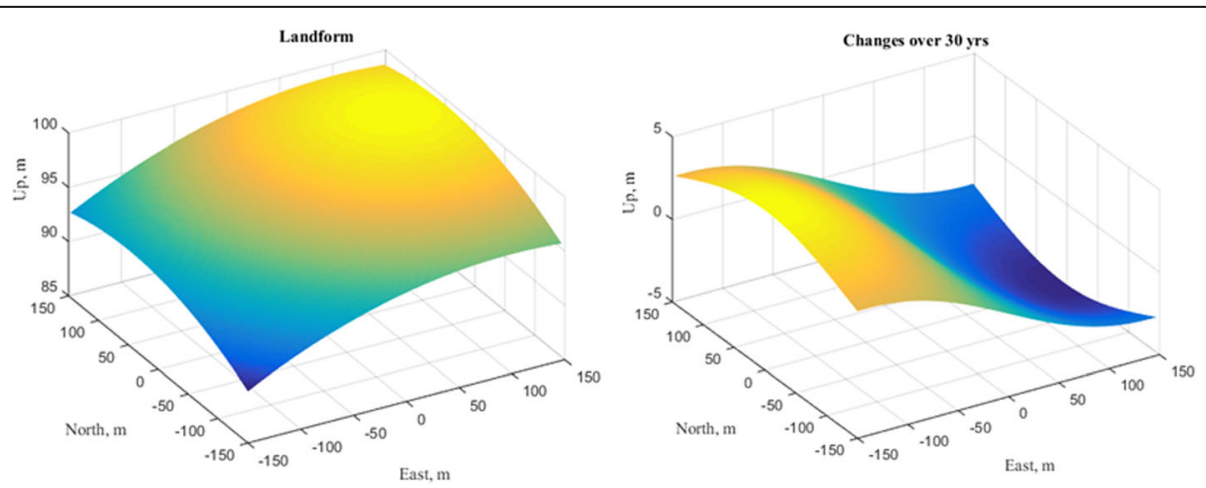

Fig. 1 Simulated landform (left) and accumulated changes over 30 years (right). The magnitude of change is approximately $\pm 5 \mathrm{~m}$ 
Table 1 Sensor resolution and error

\begin{tabular}{lllllll}
\hline Sensor name & TLS & ALS & 2D camera & SfM & Static GNSS & Kinematic GNSS \\
\hline Vertical error standard deviation in simulated data $(\mathrm{m})$ & 0.02 & 0.1 & N/A & 3.3 & 0.04 & 0.08 \\
Sample Density $\left(\mathrm{pt} / \mathrm{km}^{2}\right)$ & $1.1 \times 10^{7}$ & $7 \times 10^{3}$ & $1.1 \times 10^{5}$ & $1.1 \times 10^{5}$ & 278 & $2.2 \times 10^{4}$
\end{tabular}

The values presented herein are representative of our instrumentation and our experience to represent sensor quality. The large vertical error is observed with SfM since the displacement between camera perspectives is short in the simulation and is represented of legacy aerial photography. Sample density represents the density of measurements used to update the estimator in this simulation. It does not necessarily represent the density of raw data available from these sensors (such as TLS and ALS)

cloud, and has more accurate ranging measurements. The ALS, on the other hand, scans the ground from a high altitude, in which case the vertical error is less sensitive to the angular uncertainty.

Data from a 2D camera image does not provide direct observation of elevation, as can be seen in Table 1. However, 2D images can be related to the 3D landform via a 3D-2D projection model (Hartley and Zisserman 2003). Let vector $[u, v]^{T}$ represent the $2 \mathrm{D}$ location of a point $L$ on the image of a landform, observed using a camera with lens focal length $f$, and vector $\left[x_{L}^{C}, y_{L}^{C}, z_{L}^{C}\right]^{T}$ be the $3 \mathrm{D}$ location of point $L$, as observed in the camera frame (C frame). The 3D-2D relationship can be modeled with

$$
\left[\begin{array}{l}
u \\
v
\end{array}\right]=\frac{f}{z_{L}^{C}}\left[\begin{array}{c}
x_{L}^{C} \\
y_{L}^{C}
\end{array}\right]
$$

which is implemented in the estimator. Any change in $x_{L}^{C}$ and $y_{L}^{C}$ are thus observable in $2 \mathrm{D}$ images. The landform elevation change is observed on the $z$ axis in a Global frame $G, \Delta z_{L}^{G}$. As shown in Fig. 3, point $L$ is located at $\boldsymbol{X}_{L}^{G}$, and it is being observed by a camera located at $X_{C}^{G}$, both in the global frame. The elevation error $\Delta z_{L}^{G}$ is related to the observation error made in the camera frame $\Delta x_{L}^{C} . \Delta x_{L}^{C}$ can be geometrically decomposed into errors in the horizontal direction, $\Delta x_{L}^{G}$ and the vertical direction, $\Delta z_{L}^{G}$ in the global frame. The camera associated with the aerial photography is typically located above the landform (Fig. 3). The vertical observable $\Delta z_{L}^{G}$ is thus smaller than the horizontal component $\Delta x_{L}^{G}$ in this geometry. Therefore, it tends to be less effective
(Table 1) when elevation changes are detected with an overhead image collected with an airborne camera.

The aforementioned projection model should not be confused with SfM-MVS, which operates on the same principle of multi-view geometry (Hartley and Zisserman 2003), as does stereo vision or triangulation. A camera in motion (with a limited overlapping nadir-looking images to simulate a historic aerial photogrammetric approach) will provide repeated observations of the same 3D landform $[u, v]_{1}^{T} \ldots n$, collected from $n$ perspectives (unstructured photographs). In this process, the camera orientation and location are known at all $n$ perspectives, referenced to frame $G$. The $2 \mathrm{D}$ images, $[u, v]_{1 .}^{T}$. n will then be converted into unit vectors in frame $G, \boldsymbol{U}_{1 .}^{G} . n$. At the $i$ th perspective, $\boldsymbol{U}_{i}^{G}$ points from the camera position $\boldsymbol{X}_{C, i}^{G}$ to the landform position $\boldsymbol{X}_{L}^{G}$. Since $\boldsymbol{X}_{L}^{G}$ does not change over the different perspectives, it is solved by using $\boldsymbol{U}_{1 .}^{G} . n$ and $\boldsymbol{X}_{C, 1 . . n}^{G}$ via an optimization process (Hartley and Zisserman 2003). Figure 4 illustrates the geometric relationship between the landform and the camera on perspectives 1 and $n$. The uncertainty of $\boldsymbol{X}_{L}^{G}$ will also be dependent on the accuracy of $\boldsymbol{U}_{1 .}^{G}$. $n$ and $\boldsymbol{X}_{C, 1 . . n}^{G}$, and the geometric relationship between $\boldsymbol{X}_{C, 1 . . n}^{G}$ and $\boldsymbol{X}_{L}^{G}$. We conservatively assume a short displacement between camera perspectives in this experiment, which results in greater vertical errors in SfM.

The stochastic estimator uses measurements that become available sequentially over time. Let a state vector $x$ represent the vertical errors on the 25 anchors, located at $\boldsymbol{m}_{x[1 . .25]}$, and a 25 by 25 matrix $\boldsymbol{P}$ for the covariance among states. $\boldsymbol{x}$ and $\boldsymbol{P}$ are initialized with some

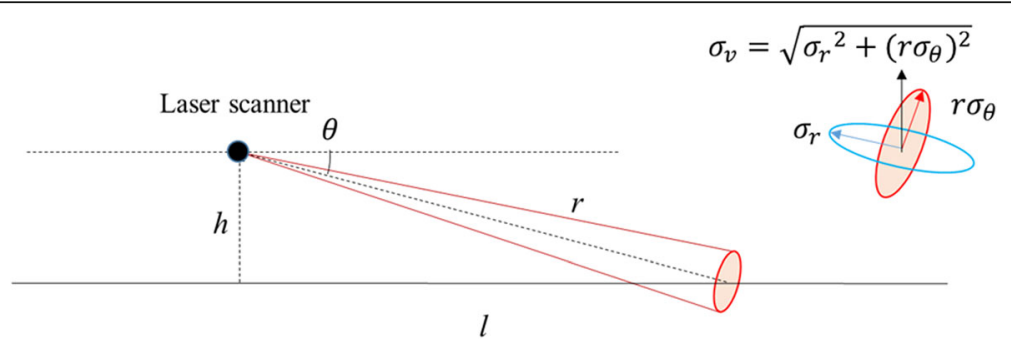

Fig. 2 Laser scanner error model. $\theta$ : vertical angle, $r$ : range, $\sigma_{r}$ : standard deviation of radial ranging error, $\sigma_{\theta}$ : standard deviation of vertical angular error, $\sigma_{\mathrm{v}}$ : standard deviation of total vertical error 


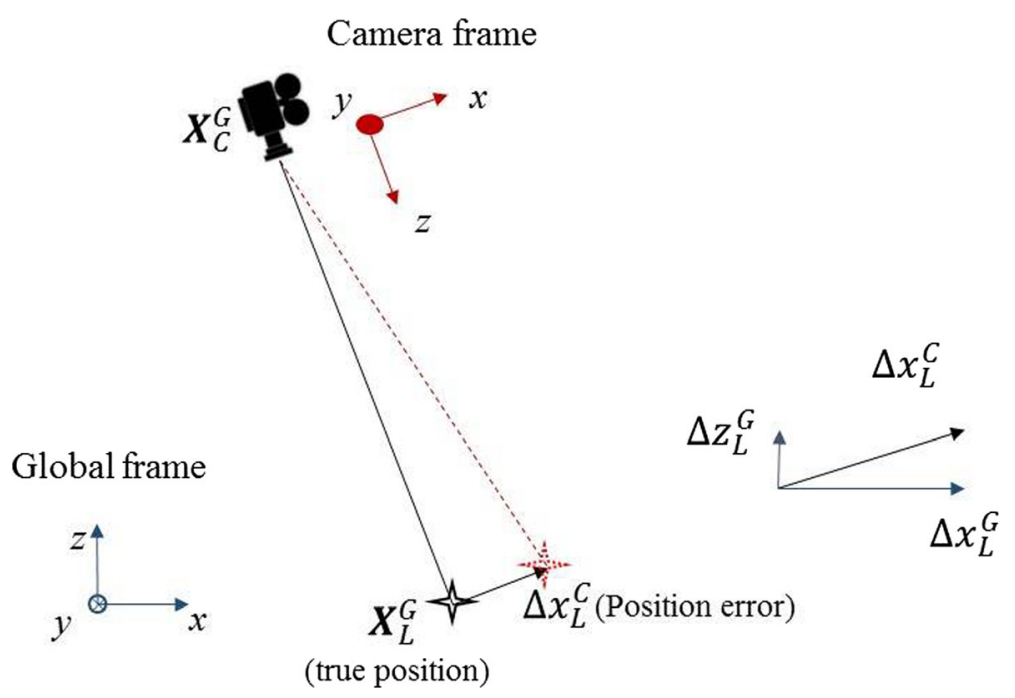

Fig. $32 \mathrm{D}$ camera image error model in a typical aerial photography application

measurements, such as a TLS scan at time $t_{k-1}$, and then propagated to another time $t_{k}$ using a Brownian process.

$$
\begin{aligned}
& \boldsymbol{x}_{\boldsymbol{k} ; \boldsymbol{k}-1}=\boldsymbol{F} \boldsymbol{x}_{\boldsymbol{k}-1 ; \boldsymbol{k}-1}+\boldsymbol{\mu} \\
& \boldsymbol{P}_{\boldsymbol{k} ; \boldsymbol{k}-1}=\boldsymbol{F} \boldsymbol{P}_{\boldsymbol{k}-1 ; \boldsymbol{k}-1} \boldsymbol{F}+\boldsymbol{Q},
\end{aligned}
$$

where $\boldsymbol{F}$ is a matrix describing the dynamic relationship between states, which will be the identity matrix in this case. $\boldsymbol{\mu}$ is the process noise component, corresponding to the Brownian process, of which the covariance matrix is defined with $Q$.

Without additional measurements, the matrix $\boldsymbol{P}$, will grow as a function of time. The Brownian process may be established with the best-known model of terrain change, and will only be used to predict the increase of uncertainty if the model is valid for the underlying geomorphic process.
The fundamental concept can be illustrated using just two of the anchor points, located at $\boldsymbol{m}_{x[1]}$ and $\boldsymbol{m}_{x[2]}$, respectively. The initial uncertainty of both points can be represented with two circles at $t_{0}$ (Fig. 5). As time propagates to $t_{1}$, the uncertainty grows for both points, illustrated with greater circles. A new measurement becomes available at $t_{1}$, made at location $\boldsymbol{m}_{z}\left[t_{1}\right]$. The uncertainty of this measurement is represented with an ellipse. This measurement is then used to update both anchor points. Right after this update, at $t_{1+}$, the uncertainty of both points is reduced to ellipses. Since the update is closer to $\boldsymbol{m}_{x[2]}$, the corresponding ellipse becomes smaller than that of $\boldsymbol{m}_{x[1]}$. Without additional measurements, both ellipses are propagated to $t_{2}$, which result in greater ellipses. A second measurement was made at $t_{2}$, which occurred much closer to $\boldsymbol{m}_{x[1]}$ this time. It effectively reduced the size of the ellipses, more so on $\boldsymbol{m}_{x[1]}$ than $\boldsymbol{m}_{x[2] \text {. }}$
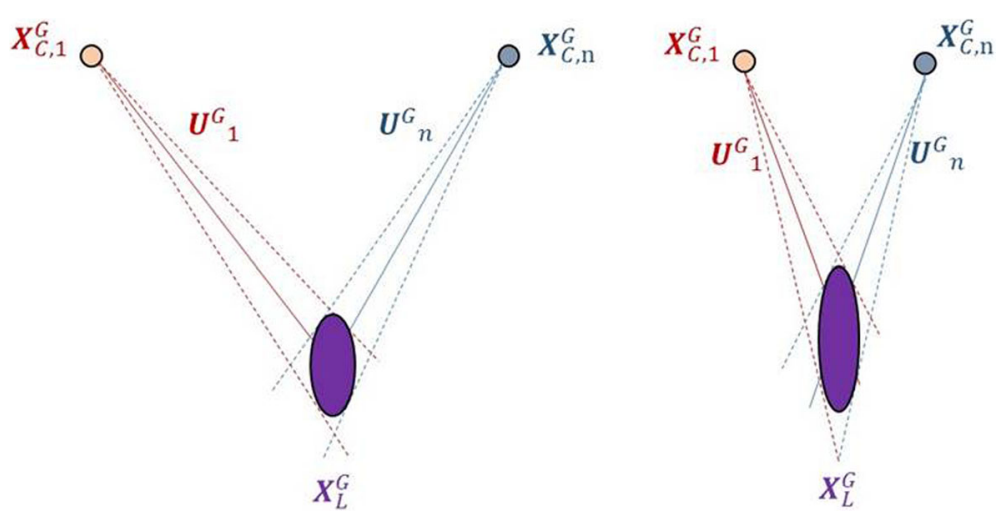

Fig. $4 \mathrm{SfM}$ error model with two perspectives. A long displacement example is shown on the left, with the error of landmark location illustrated in a purple ellipse. On the right is a short displacement example, where the error is greater in the vertical direction 


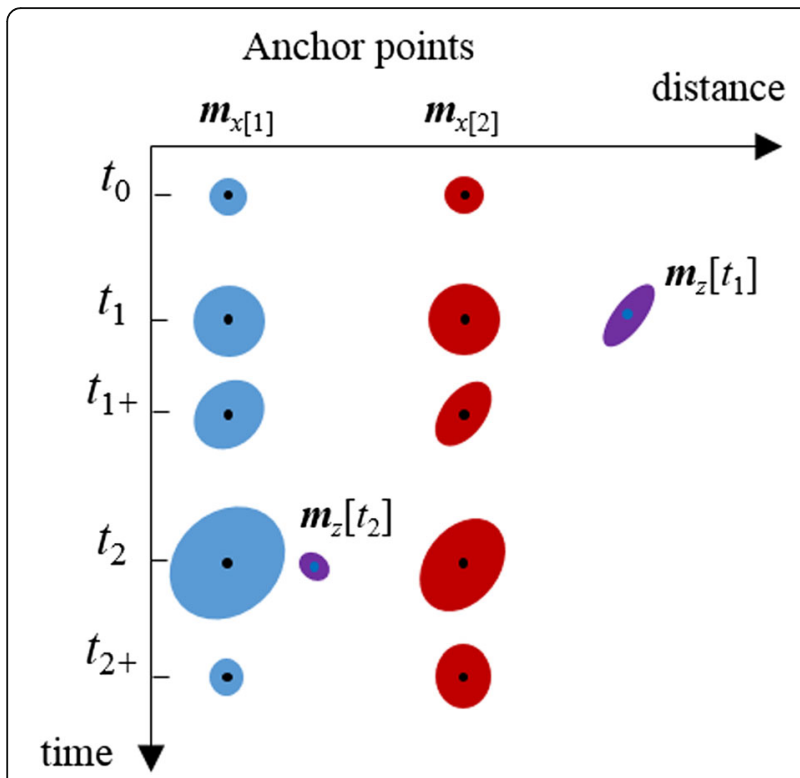

Fig. 5 Two anchor points located $\mathrm{mx}_{[1]}$ (b/ue) and $\mathrm{mx}_{[2]}$ (red) show what happens when new measurements become available over time. Uncertainty in both points are represented by circles and the circles increase and decrease in size with increasing or decreasing uncertainty, respectively

The geometric relationship between anchor points and any measurement can still be represented or approximated with a linear/linearized model, which can be established by using an interpolation method. Notice that the interpolation model used in this step estimates the covariance function, instead of predicting the landform as does Kriging or polynomial regression. The bicubic interpolation method introduced in Keys (1981) has been widely used in the computer vision community, and it has been adopted in digital elevation surface modeling (Dumitru et al. 2013). With bicubic interpolation, a linearized relationship between a given point and the anchor points, denoted with matrix $\boldsymbol{H}$, can be easily estimated. This approach handles non-linear landforms, and remains relatively computationally efficient.

The elevation at $\boldsymbol{m}_{\boldsymbol{z}}$ now has a direct measurement $\boldsymbol{z}$, and a prediction from the estimator states, $\boldsymbol{H x}_{\boldsymbol{k} ; \boldsymbol{k}-1}$. Any disagreement between them is likely a result of uncertainties associated with both and new information that can be observed as "innovation" on the states,

$$
\boldsymbol{y}=\boldsymbol{z}-\boldsymbol{H} \boldsymbol{x}_{\boldsymbol{k} ; \boldsymbol{k}-1},
$$

with a de-facto standard estimation method, the Kalman filter (Kalman 1960), $\boldsymbol{x}$ and $\boldsymbol{P}$ are updated with a gain $\boldsymbol{K}$ :

$$
\boldsymbol{K}=\boldsymbol{P}_{\boldsymbol{k} ; \boldsymbol{k}-1} \boldsymbol{H}^{\prime}\left(\boldsymbol{H} \boldsymbol{P}_{\boldsymbol{k} ; \boldsymbol{k}-1} \boldsymbol{H}^{\prime}+\boldsymbol{R}\right)^{-1}
$$

which allows us to update the states $\boldsymbol{x}$ and covariance $\boldsymbol{P}$ using this new information:

$$
\begin{aligned}
& \boldsymbol{x}_{\boldsymbol{k} ; \boldsymbol{k}}=\boldsymbol{x}_{\boldsymbol{k} ; \boldsymbol{k}-1}+\boldsymbol{K} \boldsymbol{y} \\
& \boldsymbol{P}_{\boldsymbol{k} ; \boldsymbol{k}}=(\boldsymbol{I}-\boldsymbol{K} \boldsymbol{H}) \boldsymbol{P}_{\boldsymbol{k} ; \boldsymbol{k}-1}
\end{aligned}
$$

After this step, the states are further propagated onto time $\boldsymbol{t}_{\boldsymbol{k}+1}$, with the integration of another measurement (such as aerial photo). The time history of states $\boldsymbol{x}$ and covariance matrix $\boldsymbol{P}$ are the outcome of this approach. Notice that only a few key steps of the estimator are highlighted here for the sake of conciseness. For details on the derivation and implementation, please refer to (Kalman 1960) and (Smith et al. 1962).

At any given point in time, $\boldsymbol{x}$ and $\boldsymbol{P}$ are used as input to predict the elevation and uncertainty of any given point on the landform with a standard interpolation method. An accurate and consistent covariance matrix $\boldsymbol{P}$ is the key to an optimum interpolation process. Consistency of covariance is defined based on how well $\boldsymbol{P}$ describes and over-bounds the actual error in states $\boldsymbol{x}$. To verify the estimation, $\boldsymbol{x}$ will be compared against a truth reference $\boldsymbol{x}_{\boldsymbol{r} \text { ef }}$ used in the software simulation. With field data, the truth reference can be generated from a high-resolution, high-fidelity sensor such as TLS. The variance and covariance of error $\Delta \boldsymbol{x}=\boldsymbol{x}-\boldsymbol{x}_{\text {ref }}$; is expected to be closely bounded by $\boldsymbol{P}$ at each time step $t_{k}$. Since it may be difficult to visualize the comparison between matrices, the standard error $\sigma_{\Delta x}[\boldsymbol{k}]$ will be compared against an uncertainty level for this step, by using the root mean square of all the diagonal elements in $\boldsymbol{P}$.

$$
\overline{\boldsymbol{\sigma}}_{\text {est }}[\boldsymbol{k}]=\sqrt{\overline{\operatorname{diag}\left(\boldsymbol{P}_{\boldsymbol{k} ; \boldsymbol{k}}\right)}}
$$

In the software simulation, the actual elevation change is not provided to the estimator. Rather, an overbounding Brownian process is used, and we expect to have $\bar{\sigma}_{\text {est }} \geq \sigma_{\Delta x}$ in the history of 30 years.

\section{Physical modeling experiment}

A physical model is developed in the Terrain Analysis $\mathrm{Lab}$ at East Carolina University as a further means of validating our uncertainty measures. The controlled simulation is designed around a fan-shaped surficial features (i.e. an alluvial fan or washover fan) built in a stainless-steel stream table ( $3.7 \mathrm{~m}$ long by $1.8 \mathrm{~m}$ wide) using coarse sand on a white sheet of plotter paper to reduce reflectivity of the stainless-steel table (Fig. 6). The fan-shaped feature is $0.974 \mathrm{~m}$ wide and $0.392 \mathrm{~m}$ in with a relief of $0.05 \mathrm{~m}$ from the bottom of the stream table (Fig. 6).

A Leica P20 laser scanner was inverted and mounted on an aluminum swing set (Lisenby et al. 2014). Targets mounted on the wall of the lab and within the stainlesssteel table as well as the corner points of the table were used to register the TLS data (Fig. 6). Cartesian 
a

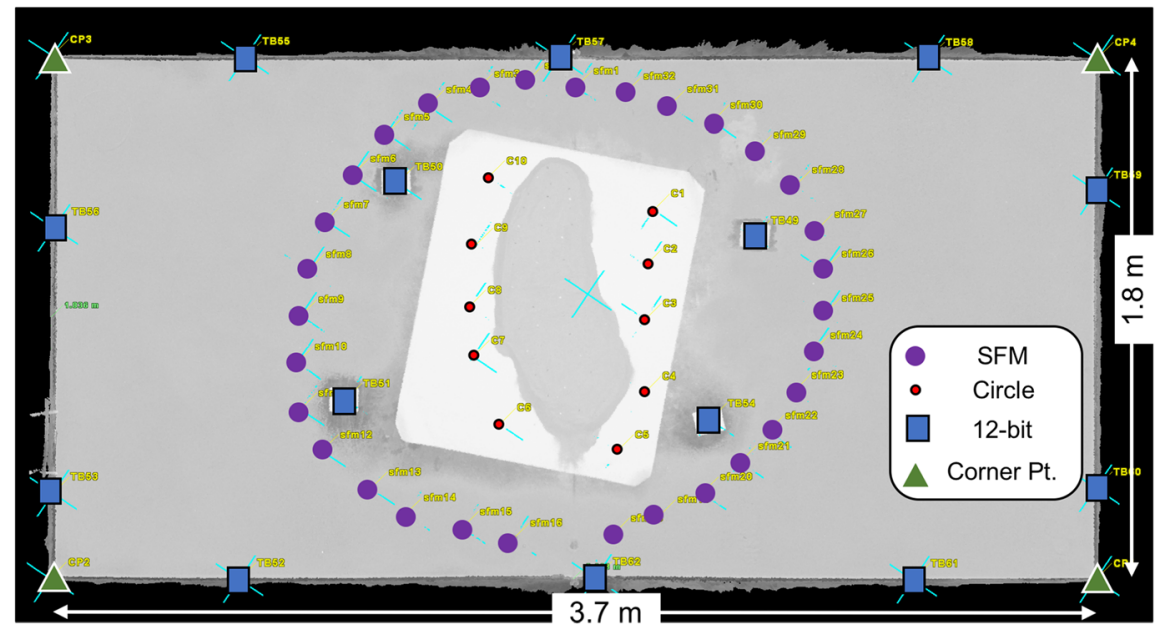

b

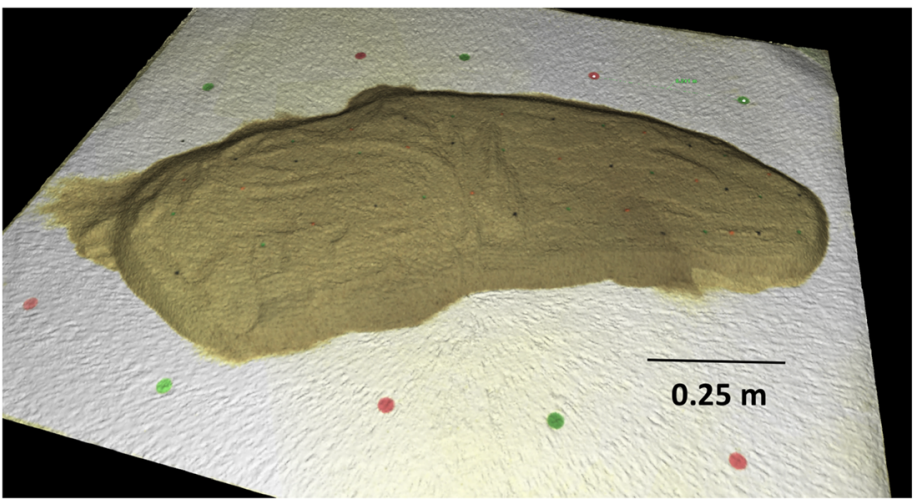

\section{C}

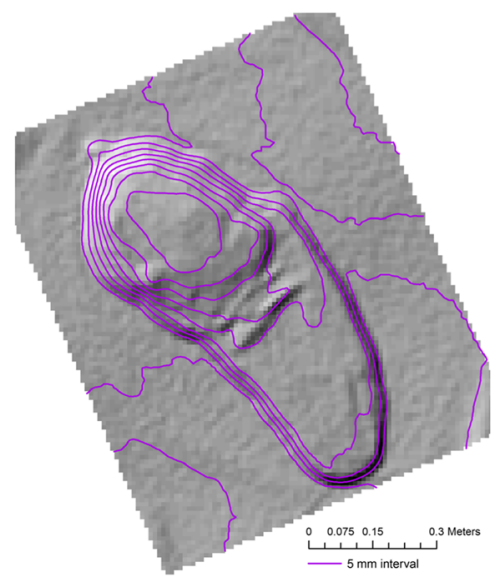

d

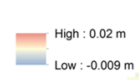

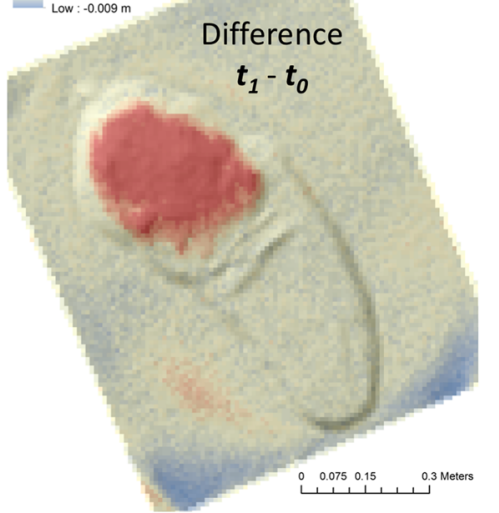

Fig. 6 The experimental fan model. a Location of control points and cameras. $\mathbf{b}$ SfM image of the fan on the plotter paper with the circle targets (image rotated $90 \mathrm{CCW}$ to A). $\mathbf{c}$ A topographic map of the experimental fan. $\mathbf{d}$ Elevation difference from $t_{1}$ to $t_{0}$

coordinates associated with TLS point clouds provide locations for the control points used in the SfM. A single scan from the nadir looking position of the scanner captured the entire simulated fan surface in $t_{0}$ and $t_{1}$. SfM data were collected with a Ricoh GR II digital camera. The camera was positioned at two different heights at 32 locations looking obliquely and inward at the fan surface (Fig. 6). A total of 64 images were used to generate a point cloud with the aid of Agisoft Photoscan software. A combination of 14 12-bit targets from the Agisoft software were printed on sticky back paper and adhered to the stainless-steel table, along with 10 small circle targets with known dimensions. Targets and circles were used to aid in the photo alignment process.

The original landform $\left(t_{0}\right)$ was modified $\left(t_{1}\right)$ by adding approximately $2 \mathrm{~cm}$ of coarse sand onto a segment of 
the fan (Figs. 6 and 7) to emulate fan segment aggradation over an arbitrary time-period (for example, $t_{1}$ is set to 30 years). TLS and SfM-MVS data are collected at both $t_{0}$ and $t_{1}$. TLS at $t_{1}$ is used as a truth reference. We initialize the estimator at $t_{0}$ with TLS and SfM-MVS, propagate and update at $t_{1}$ using SfM-MVS. The estimated $\bar{\sigma}_{\text {est }}[1]$ is compared against the actual error $\sigma_{\Delta x}[1]$ in this case.

\section{Results and discussion}

The estimator in the software simulation is initialized with a TLS scan. Subsequently, five additional sensor data sets become available over 30 years, to observe the geomorphic change. A general pattern evolves whereby the estimated uncertainty provides an over bound of the actual error (Fig. 8), i.e., $\bar{\sigma}_{\text {est }} \geq \sigma_{\Delta x}$. Both $\bar{\sigma}_{\text {est }}$ and $\sigma_{\Delta x}$ grow over time during the simulation, and are dramatically reduced by some measurements, such as ALS, SfMMVS and Kinematic GNSS surveys. Other sensors, such as 2D photos and static GNSS, are less effective, as expected. Although the SfM-MVS measurements are not nearly as accurate as static GNSS survey, it offers a much higher point density and therefore, is also able to reduce the error more effectively. The over-bounding associated with the static GNSS has more to do with the lack of data produced with this technique than the accuracy of the data collected. The truth reference used in this software simulation can be used to verify the estimation in $\boldsymbol{x}$ (Fig. 9).

The estimator is also applied to the changes associated with the lab-simulated fan model. As this terrain is more sophisticated, a 20 by 20 mesh of anchors is used. The actual change $\boldsymbol{x}_{\boldsymbol{r} e f}$ is obtained by comparing the two TLS scans (Fig. 10). The estimated change is observed on the anchor points, by using a less accurate and lowerresolution SfM-MVS update. At $t_{1}=30$ years, $\bar{\sigma}_{\text {est }}=5.6$ $m m$ and $\sigma_{\Delta x}=3.7 m m, \bar{\sigma}_{\text {est }}$ is greater than, and yet reasonably close to $\sigma_{\Delta x}$. The difference is below the detectable limits for topographic change associated with the data source instrumentation (Wester et al. 2014, Staley et al. 2014; Wasklewicz and Scheinert 2015).

The software simulation and physical modeling experiment both show that the estimator can provide a reliable and consistent measure of uncertainty in legacy data. Although some sensors are more desired than others, the availability of sensors in legacy data and sometimes also in new data collection campaigns dictates that data from all sensors must be incorporated. The estimator will allow us to retrieve information from sensors of low accuracy, low observability (2D images) or low spatial resolution (static surveys). We further emphasize that the discussed estimator is not limited to just the Kalman filter. Other Bayesian estimators, such as particle filters (Montemerlo and Thrun 2003), are applicable as well. Similarly, bicubic interpolation is not the only choice for interpolation either. Other methods that can yield a linearized relationship between measurements and states are also valid options, which will provide various types of advantages (Dumitru et al. 2013).

The statistical consistency of uncertainties in all the sensor measurements and estimates are critical in our application. In practice, repeated measurements could also lead to a covariance matrix $\boldsymbol{P}$ that is too small (Julier and Uhlmann 2001), such as high-density data from SfM-MVS. As $\boldsymbol{x}$ and $\boldsymbol{P}$ can be used to predict the elevation and uncertainty of the whole landform, an overly optimistic $\boldsymbol{P}$ can easily cause unexpected errors in the prediction. For instance, few erroneous points in $\boldsymbol{x}$

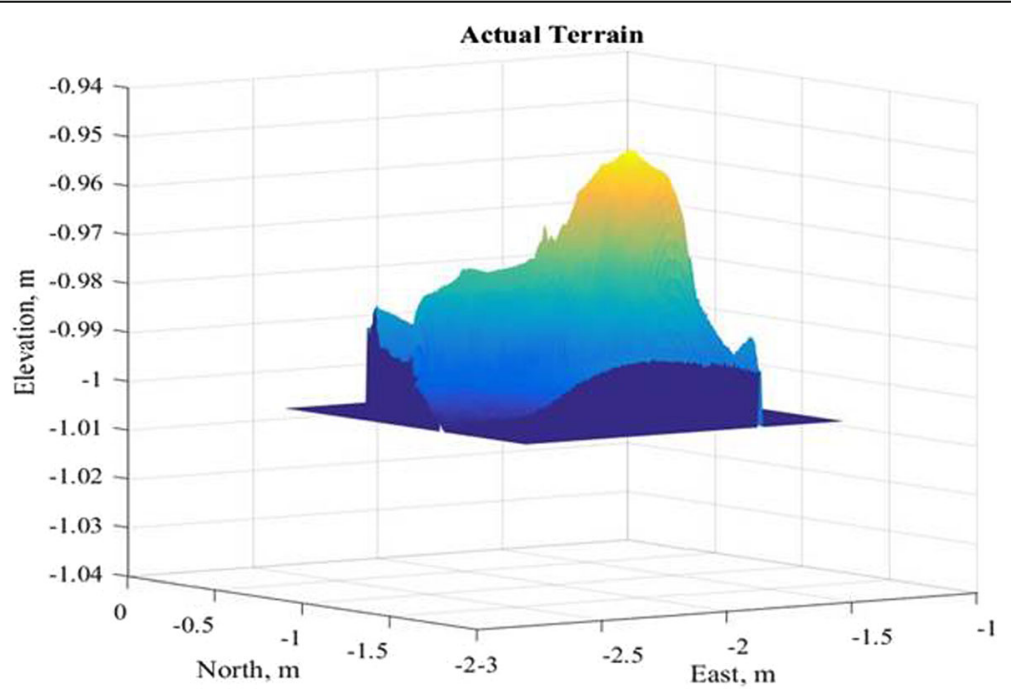

Fig. 7 Elevation model from TLS point cloud of the simulated fan model at $t_{1}$ 


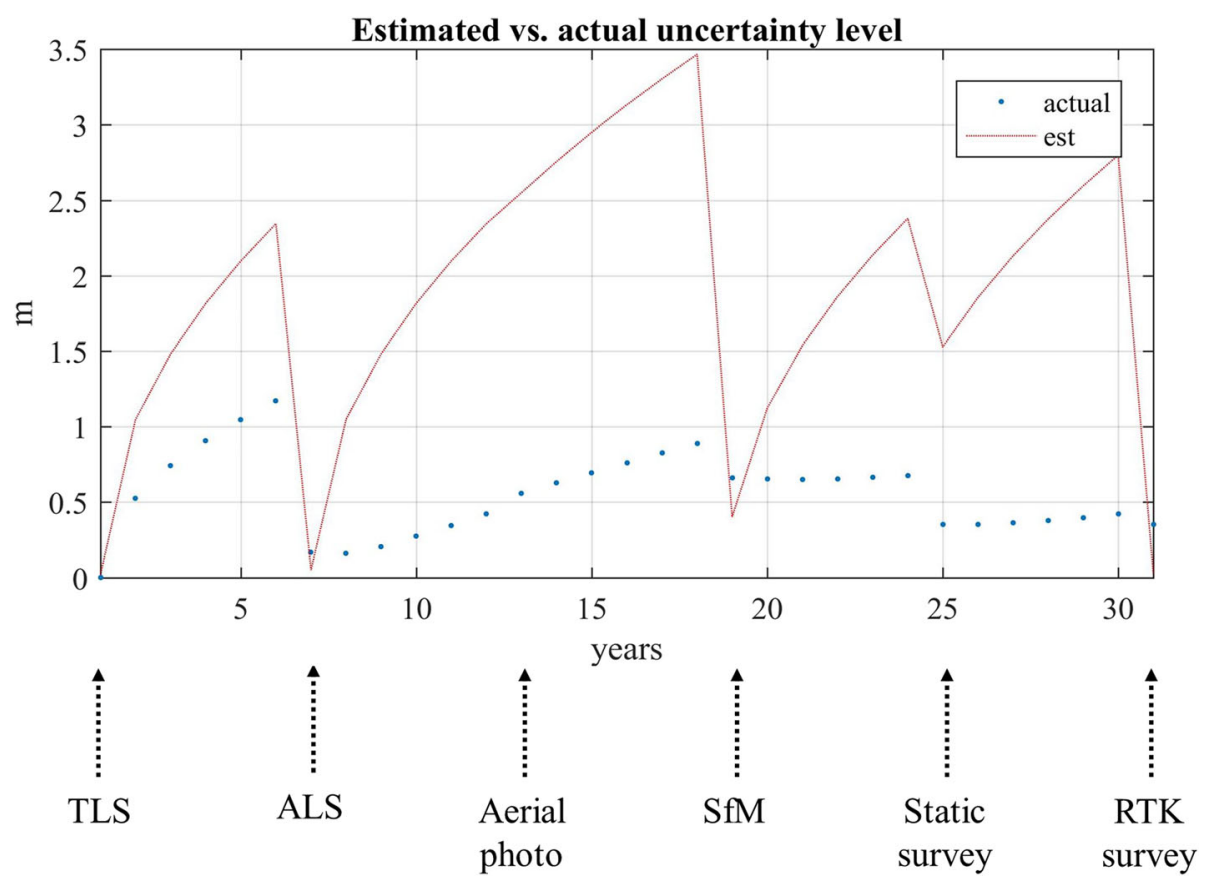

Fig. 8 Uncertainty of software simulated landform over 30 years, estimated sigma $\overline{\boldsymbol{\sigma}}_{\text {est }}$ vs. actual error $\sigma_{\Delta x}$

with small variances would dominate the prediction and generate a bias in a neighborhood. Overly optimistic $\boldsymbol{P}$ also would prevent $\boldsymbol{x}$ from being updated with other sensors. Additional steps will be taken in future work to guarantee the consistency of $\boldsymbol{P}$.

The successful outcomes and corroboration in our initial software and physical modeling simulations, the ability to adopt and use multiple estimators and interpolation techniques, and an ability to be employed across a variety of environmental settings provides a new alternative to integrating legacy and HRT data.

Applications of existing and planned methodology to field research will help extend our understanding of the dynamic landform and landscape changes that take place through space and time. At present, earth science researchers conducting studies with the aid of HRT data have placed a great deal of emphasis on methods and techniques that provide rapid, accurate, and spatially continuous topographic data. HRT provide the user with unparalleled information of short-term changes in processes and forms. Subtle topographic changes that earth scientists must measure in the future, for example those associated with climate change (Lane 2013), require detailed data that can be precisely acquired and accurately assessed to address scientific questions and inform policy makers. However, the sole use of HRT data limits the
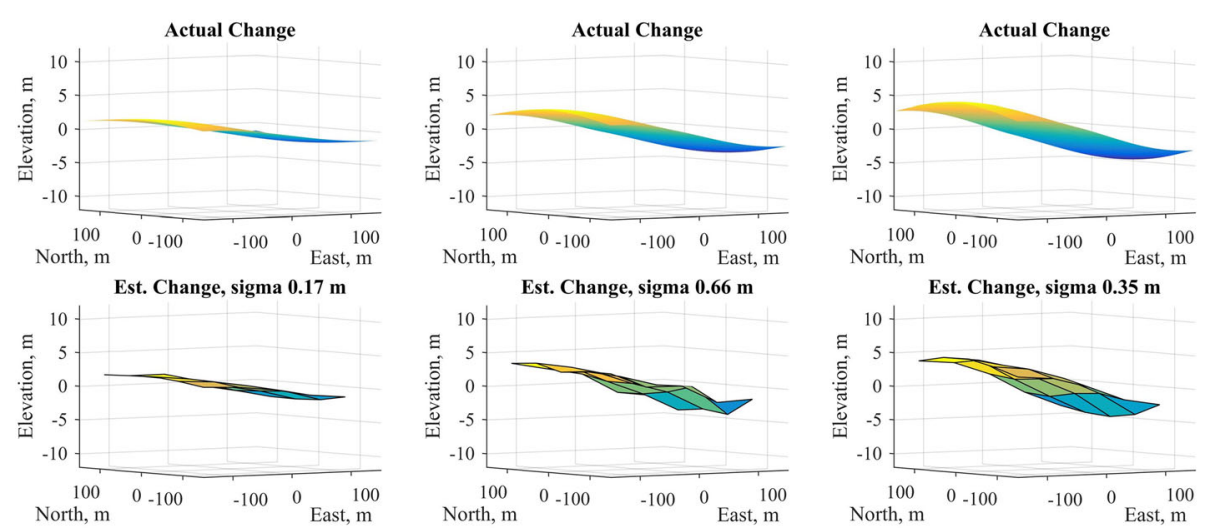

Fig. 9 Actual vs. estimated terrain change in year 7 (left), year 19 (middle) and year 31 (right). Upper: actual terrain change $\boldsymbol{x}_{\text {refi }}$ lower: estimation in states $\boldsymbol{X}$ 


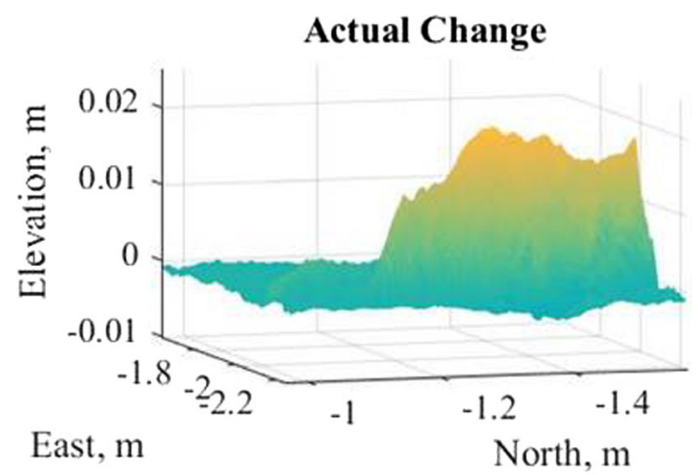

Est. Change

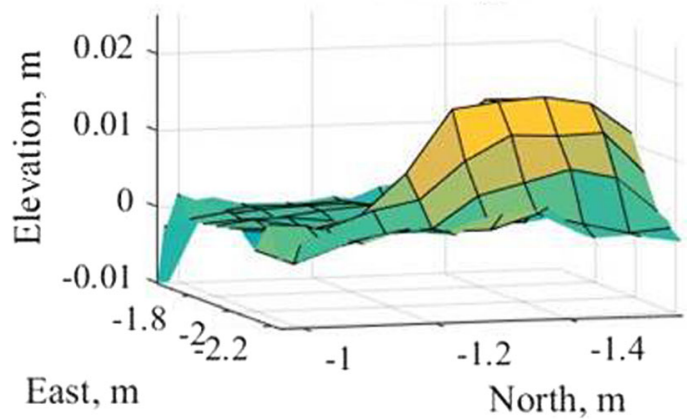

Fig. 10 Actual vs. estimated terrain change in lab-simulated fan model. Upper: actual terrain change $\boldsymbol{x}_{\text {ref, }}$ lower: estimation in states $\boldsymbol{x}$

temporal framework for which earth scientists can consider these changes because these data sets have only been recently developed. Legacy topographic data allow us to extend this temporal perspective of change backward in time to better inform us of variations on decadal time-scales and in some instances, as far back as a halfcentury or more (Corsini et al. 2009; Carley et al. 2012; James et al. 2012; Schaffrath et al. 2015). While we appreciate that this represents a miniscule amount of time from a geological perspective, the ability to use legacy topographic data increases the temporal sample size earth scientists are working from and allows us to make more authoritative statements about landform evolution than the shorter-term view supplied by research solely using HRT data.

Scientists need to be able to definitively measure the environmental changes and communicate these measures to a broad audience of possible users that include their peers, public officials, and the community. Expressing the uncertainty associated with these measurements or reducing the uncertainty in the data production process is a critical first step to achieving more definitive measures and communication. Here, our uncertainty model, associated anchor points, and stochastic estimator clearly highlight that spatial uncertainty can be accounted for across the time-scales of the legacy and HRT data. Each elevation point/grid has an associated error, which can be used to produce better maps when interpolating to raster data and provide better measurement tolerances as they relate to topographic changes and sediment transport.

The ability to accurately assess spatially variable uncertainty across a broad range of temporal scales also provides a means to exchange information between researchers, engineers, policy makers, and environmental managers and planners. The longer-term understanding of topographic change and sediment transport and the uncertainties associated with these dynamics permits greater understanding of issues such as: water quality, habitat loss, and risks to built-environments and human lives from environmental hazards. Furthermore, an ability to measure the level of data uncertainty associated with past landform and landscape dynamics will help advance landscape evolution models because of the ability to corroborate existing landscape evolutionary models with the longer-term record of topographic change derived from legacy topographic data sets and recent HRT data. This is a necessary advancement as we may come to rely on landscape evolution models as an integral tool in the decision and management schemes as we face climate change and increased scenarios of extreme weather.

\section{Conclusions}

Our software simulation and physical modeling experiments provide a new approach to measuring topographic data uncertainty where legacy data from a variety of data sources can be integrated with HRT data to expand the time-scales of topographic change detection. As anticipated, the difference in the actual and expected errors of our HRT physical model experiment was quite small $(<2 \mathrm{~mm})$. Current instrumentation and field methods often have a higher minimal level of detection, so this value is quite acceptable for HRT data sources and represents a value comparable to or lower than most uncertainty measures found in current research exploring topographic change detection. The uncertainty model, associated anchor points, and stochastic estimator was further applied to a software simulation whereby a variety of remote-sensing data sources were used to simulate data capture from legacy data sources. Our findings show the estimated error coincides with the actual error using certain sensors (Kinematic GNSS, ALS, TLS, and SfM-MVS). Data from 2D imagery and static GNSS did not perform as well at the time the sensor is integrated into estimator. Nevertheless, the software simulation shows the approach can be used to estimate the error associated with all elevation values in the legacy and HRT data over the time-period of the simulation.

Our findings show a strong potential for this technique to be applied in a variety of field settings. We anticipate further development of the uncertainty model in 
future research. Our goal is to expand the capability to densify the anchor points in areas that are more topographically complex and test by overfitting the surface how well the uncertainty model is performing in these locations. At present, it is not clear if the use of sparse points (for example, techniques that rely on interpolation from topographic maps) under sample the topographic surface and therefore, do not accurately represent the surface or the spatially variable uncertainty in the measurement of topographic change. We intend to expand the capabilities of the model by conducting further software simulations that analyze and visualize topographic change over time using the varied densification of anchor points. The advances in the uncertainty model will then be further evaluated using real-world data from a variety of sources. For example, ALS data can be used in a fashion to simulate different types of data by varying the data density, data noise, etc. to examine known differences in the data and the capability to measures these data perturbations using the refine uncertainty model. Once these items have been worked out our intent is to apply the uncertainty model to the real-world topographic and environmental changes within various environmental settings that include more topographically complex areas like mountainous environments versus less topographic complex locales such as barrier islands.

Our existing uncertainty model shows clear evidence that the temporal component of spatially variable uncertainty of legacy topographic data sets can be measured in both of our simulations. A capacity to integrate legacy topographic data expands earth scientist's ability to understand topographic changes and sediment transport over longer time-scales. This should lead to more definitive measurements and answers regarding how environments are changing over the past 50 to 100 years (dependent upon the temporal availability of topographic data sources). More conclusive measures and findings stem from our ability to assess uncertainty across the spectrum of legacy data in relation to more recent HRT data using techniques like we have documented in this research. New results from these approaches should provide valuable information to the broader scientific community and society. Societal benefits will likely be recognized in the form of delivering relevant information that show the potential range for environmental changes over time-scales demanded by managers and policy makers.

\footnotetext{
Abbreviations

ALS: Airborne laser scanning; DEM: Digital elevation model; DEM-of-difference; GNSS: Global navigation satellite system; HRT: High-resolution topography; SfMMVS: Structure from Motion-Multi-View Stereo; SIE: Survey and interpolation error; TIN: Triangular irregular networks; TLS: Terrestrial laser scanning
}

\section{Funding}

This work was funded by an East Carolina University Interdisciplinary Research Collaboration Award entitled "Development of Scalable Sensor Payload for Rapid High Resolution Topographic Scanning via Micro Aerial Vehicles". Funding for this award was supplied by the Division of Research and Graduate Studies in partnership with the Divisions of Academic Affairs and Health Sciences. The Terrain Analysis Lab and ECU Department of Geography, Planning, and Environment, provided the physical modeling facilities and equipment to simulate field measurements in a controlled setting.

\section{Authors' contributions}

TW proposed the topic, helped conceive the study design, analyzed the data, helped with the interpretation of the data, co-wrote the paper, and is corresponding author. ZZ helped conceive the study design, carried out the experimental study, analyzed the data, interpreted the data, designed figures, and co-wrote the paper. PG helped in the interpretation of the data and co-wrote the paper. All authors read and approved the final manuscript.

\section{Competing interests}

TW, ZZ, and PG declare that they have no competing interest.

\section{Publisher's Note}

Springer Nature remains neutral with regard to jurisdictional claims in published maps and institutional affiliations.

\section{Author details}

${ }^{1}$ Department of Geography, Planning, and Environment, East Carolina University, Brewster Building A227, Greenville, NC 27858, USA. ${ }^{2}$ College of Engineering and Technology, East Carolina University, Science and Technology Complex Suite 100, Greenville, NC 27858, USA.

Received: 28 January 2017 Accepted: 28 September 2017

Published online: 30 October 2017

\section{References}

Brasington J, Langham J, Rumsby B (2003) Methodological sensitivity of morphometric estimates of coarse fluvial sediment transport. Geomorphology 53:299-316

Brasington J, Rumsby BT, McVey RA (2000) Monitoring and modelling morphological change in a braided gravel-bed river using high resolution GPS-based survey. Earth Surface Processes and Landforms 25: 973-990

Carley JK, Pasternack GB, Wyrick JR, Barker JR, Bratovich PM, Massa DA, Reedy GD, Johnson TR (2012) Significant decadal channel change 58-67 years post-dam accounting for uncertainty in topographic change detection between contour maps and point cloud models. Geomorphology 179:71-88

Corsini A, Borgatti L, Cervi F, Dahne A, Ronchetti F, Sterzai P (2009) Estimating mass-wasting processes in active earth slides-earth flows with time-series of high-resolution DEMs from photogrammetry and airborne LiDAR. Nat Hazards Earth Syst Sci 9:433-439

Cortes J (2009) Distributed kriged Kalman filter for spatial estimation. IEEE Trans Autom Control 54:2816-2827

Crowell M, Leatherman SP, Buckley MK (1991) Historical shoreline change: error analysis and mapping accuracy. Journal of coastal research, 839-852

Dumitru PD, Marin P, Dragos B (2013) Comparative study regarding the methods of interpolation. In: Recent advances in geodesy and Geomatics engineering, pp 45-52

Elfe A (1989) Using occupancy grids for mobile robot perception and navigation. Computer 22:46-57

Fonstad MA, Dietrich JT, Courville BC, Jensen JL, Carbonneau PE (2013) Topographic structure from motion: a new development in photogrammetric measurement. Earth Surf Process Landf 38:421-430

Glennie C (2007) Rigorous 3D error analysis of kinematic scanning LIDAR systems. J Appl Geodesy 1:147-157

Glennie CL, Hinojosa-Corona A, Nissen E, Kusari A, Oskin ME, Arrowsmith JR, Borsa A (2014) Optimization of legacy lidar data sets for measuring near-field earthquake displacements. Geophys Res Lett 41:3494-3501

Gomez C, Hayakawa Y, Obanawa H (2015) A study of Japanese landscapes using structure from motion derived DSMs and DEMs based on historical aerial photographs: new opportunities for vegetation monitoring and diachronic geomorphology. Geomorphology 242:11-20

Hartley R, Zisserman A (2003) Multiple view geometry in computer vision. Cambridge University Press, Cambridge 
Heritage GL, Milan DJ, Large AR, Fuller IC (2009) Influence of survey strategy and interpolation model on DEM quality. Geomorphology 112:334-344

James LA, Hodgson ME, Ghoshal S, Latiolais MM (2012) Geomorphic change detection using historic maps and DEM differencing: the temporal dimension of geospatial analysis. Geomorphology 137:181-198

Julier SJ, Uhlmann JK (2001) General decentralized data fusion with covariance intersection (CI). In: Handbook of data fusion. CRC Press, Boca Raton

Kalman RE (1960) A new approach to linear filtering and prediction problems. J Basic Eng 82:35-45

Keys R (1981) Cubic convolution interpolation for digital image processing. IEEE Trans Acoust Speech Signal Process 29:1153-1160

Krige DG (1951) A statistical approach to some mine valuations and allied problems at the witwatersrand. Master's thesis. University of Witwatersrand, Witwatersrand

Lane SN (2013) 21st century climate change: where has all the geomorphology gone? Earth Surf Process Landf 38:106-110

Lane SN, Westaway RM, Murray-Hicks D (2003) Estimation of erosion and deposition volumes in a large, gravel-bed, braided river using synoptic remote sensing. Earth Surf Process Landf 28:249-271

Lisenby PE, Slattery MC, Wasklewicz TA (2014) Morphological organization of a steep, tropical headwater stream: the aspect of channel bifurcation. Geomorphology 214:245-260

Mardia KV, Goodall C, Redfern EJ, Alonso FL (1998) The Kriged Kalman filter. TEST 7:217-285

Micheletti N, Lane SN, Chandler JH (2015) Application of archival aerial photogrammetry to quantify climate forcing of alpine landscapes. Photogramm Rec 30:143-165

Milan DJ, Heritage GL, Hetherington D (2007) Application of a 3D laser scanner in the assessment of erosion and deposition volumes and channel change in a proglacial river. Earth Surface Processes and Landforms 32:1657-1674

Milan DJ, Heritage GL, Large AR, Fuller IC (2011) Filtering spatial error from DEMs: implications for morphological change estimation. Geomorphology, 125: 160-171

Montemerlo M, Thrun S (2003) Simultaneous localization and mapping with unknown data association using FastSLAM. Proceedings of IEEE International Conference on Robotics and Automation, Taipei

Oskin ME, Arrowsmith JR, Corona AH, Elliott AJ, Fletcher JM, Fielding EJ, Gold PO, Garcia JJG, Hudnut KW, Liu-Zeng J, Teran OJ (2012) Near-field deformation from the el mayor-Cucapah earthquake revealed by differential LIDAR. Science 335:702-705

Pelletier JD, Brad Murray A, Pierce JL, Bierman PR, Breshears DD, Crosby BT, Ellis M Foufoula-Georgiou E, Heimsath AM, Houser C., Lancaster, N (2015) Forecasting the response of Earth's surface to future climatic and land use changes: A review of methods and research needs. Earth's Future, 3(7)220-251

Schaffrath KR, Belmont P, Wheaton JM (2015) Landscape-scale geomorphic change detection: quantifying spatially variable uncertainty and circumventing legacy data issues. Geomorphology 250:334-348

Smith GL, Schmidt SF, McGee LA (1962) Application of statistical filter theory to the optimal estimation of position and velocity on board a circumlunar vehicle. National Aeronautics and Space Administration, Washington, D.C

Spencer T, Naylor L, Lane S, Darby S, Macklin M, Magilligan F, Möller I (2017) Stormy geomorphology: an introduction to the special issue. Earth Surf Process Landf 42:238-241

Staley DM, Wasklewicz TA, Kean JW (2014) Characterizing the primary material sources and dominant erosional processes for post-fire debris-flow initiation in a headwater basin using multi-temporal terrestrial laser scanning data. Geomorphology 214:324-338

Tarolli P, Sofia G (2016) Human topographic signatures and derived geomorphic processes across landscapes. Geomorphology 255:140-161

Thrun S, Burgard W, Fox D (2005) Probabilistic robotics. MIT press, Boston

Wasklewicz T, Scheinert C (2015) Development and maintenance of a telescoping debris flow fan in response to human-induced fan surface channelization, Chalk Creek valley natural debris flow laboratory, Colorado, USA. Geomorphology 252:51-65

Wasklewicz T, Staley DM, Reavis K, Oguchi T (2013) Digital terrain modeling. In: Shroder J, Bishop MP (eds) Treatise on geomorphology, vol 3. Academic Press, San Diego, pp 130-161

Wasklewicz TA, Hattanji T (2009) High-resolution analysis of debris flow-Induced Channel changes in a headwater stream, Ashio Mountains, Japan. Prof Geogr 61:231-249
Wester T, Wasklewicz T, Staley D (2014) Functional and structural connectivity within a recently burned drainage basin. Geomorphology 206:362-373

Wheaton JM, Brasington J, Darby SE, Sear DA (2010) Accounting for uncertainty in DEMs from repeat topographic surveys: improved sediment budgets. Earth Surf Process Landf 35:136-156

Williams CKI (1998) Prediction with Gaussian processes: from linear regression to linear prediction and beyond. Learn Graphical Models 89:599-621

\section{Submit your manuscript to a SpringerOpen ${ }^{\circ}$ journal and benefit from:}

- Convenient online submission

- Rigorous peer review

- Open access: articles freely available online

- High visibility within the field

- Retaining the copyright to your article

Submit your next manuscript at $\gg$ springeropen.com 